\title{
Systematic Literature Reviews and Undergraduate Research
}

A recent addition to our capstone project options in the Bachelor of Computer and Information Sciences has been the opportunity for students to undertake reviews of the research literature, as opposed to the more traditional projects involving developing a software artefact of some form. This offers an alternative for those students taking less software intensive majors or who have more of an interest in research. These projects involve conducting a full systematic review on a topic of interest.

Systematic Literature reviews are a common research approach within the software engineering discipline, and are required to adhere to well defined protocols cf. [1]. As noted in [2]

"Systematic review guidelines [1] recommend the following steps:

1. Identify the need for a systematic literature review.

2. Formulate review research question(s).

3. Carry out a search for relevant studies.

4. Assess and record the quality of included studies.

5. Classify data needed to answer the research question(s).

6. Extract data from each included study.

7. Summarize and synthesize study results (meta-analysis).

8. Interpret results to determine their applicability.

9. Write-up study as a report".

We require that a project sponsor be identified who has a need for a review with a suitable scope to be considered as a candidate project. Good candidates for a literature review are well thought through, address one or more clear research questions and are soundly framed. Possible extensions to this model involving a more practical component, could involve undertaking simulation experiments or replications of prior studies for which the data or software could be readily obtained. As with any capstone project, any proposed literature review projects need to gain approval through our normal project vetting process.

A thoroughly conducted systematic review can be time consuming and challenging, even for more senior students and researchers. Therefore an active level of supervisory guidance must be provided for undergraduate students to help scaffold the learning. In practice the systematic review will often take on the broader survey-like aspects of a systematic mapping study [3,4], through classifying and thematically analysing the literature on a selected topic. The initial research question as noted in [4] may also be somewhat generic and the focus may be on research trends (such as which researchers, how much activity, what type of studies etc.). Thus a limited mapping study could form the first stage of the review, followed by a more focused review component driven by more specific research questions, in order to offer a manageable project for the student(s). While normally this model suits an individual project, we have conducted some reviews in pairs.

We have found it useful to follow a structured process for the review, as outlined in figure 1 from Jalali \& Wohlin [3] below. 


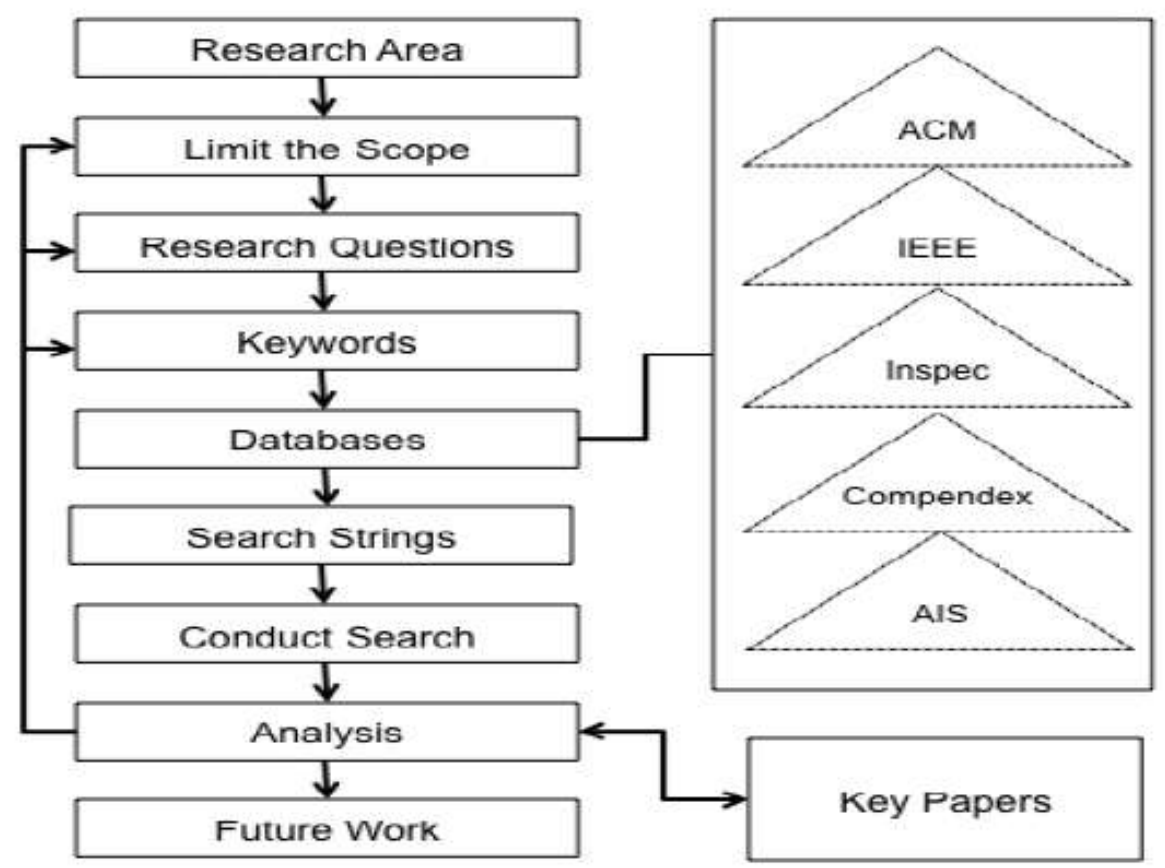

Figure 1: Search strategy and Process (ex. [3 p. 47])

This tightly structured process gives good guidance to students, and we have found for students with English as an additional language (EAL), or students without a record of high achievement in their prior courses, it helps break down the task into more manageable chunks. These can progressively produce demonstrable intermediate results, prior to the challenging final analysis and synthesis in which the findings and the implications of the review are drawn together.

As part of the final package which includes a draft article for publication delivered to their client, we have also required the progressive compilation of an annotated bibliography [5] (and accompanying Endnote ${ }^{\mathrm{TM}}$ or Bibtex reference file), to capture students' reviews of the selected articles. This output directed activity helps both students and supervisors to track the progress of their reading, and to produce discrete and measurable deliverables as the process continues. These summary entries "must have substance" and should "synthesize the contents in a concise paragraph" [5]. A suggested length for an entry is between one and two pages, but this is flexible, for instance in [5] it is recommended that: "annotations should be at least one half-page in length".

These entries can then be used by students to assist in the later synthesis process for their review. Supervisors can use them to give regular feedback to students and aid them to develop their critical faculties. The assessment rubric for an article summary/bibliography entry includes a set of simple "yes/no" questions relating to content, critique and presentation. For instance content related questions include: "concise summary - yes /no?" and "key points and findings without obvious omissions?" Questions demanding critique include: "areas of particular strength or weakness?" and "any significant omissions?" Presentation questions include "coherence and structure of critique?" and "referencing appropriate and relevant?"

From experience in applying the rubric as a diagnostic tool with research students studying at Master's level, it helps to diagnose their ability to critically review an article. For instance, a common pattern is a 'yes' for 'key points and findings', indicating the ability to describe the contents of an academic paper. By contrast often we see a pattern of several 'no's for the more critical perspectives evidenced by: identifying strengths and weaknesses; deficiencies in methodology; significant omissions; and subsequent developments. This enables a criterion 
focused discussion with students about the level of evaluation beyond description that is required to produce a critical review of an article. A quality judgement question, relating to "evidence for authority of source?" has been added to encourage a critical evaluation of the article under review for a bibliography entry. This rubric is still experimental at undergraduate level, but we have found that it provides a useful checklist for students to consider when writing their entry/review, and aids formative feedback over the depth of critical analysis and the quality of their work.

In conclusion, we have found that systematic literature reviews offer an acceptably challenging yet well structured research option for our capstone projects, they offer flexibility for single students who might not be suited to a team environment and they have proven successful for sponsors, supervisors and students. Raising the final submissions to a standard suitable for publication is our next challenge.

\section{References}

[1] Kitchenham, B. Procedures for Performing Systematic Reviews. Technical Report TR/SE-0401 NICTA 04000111T.1, National ICT Australia Ltd, Software Engineering Group, Department of Computer Science, Keele University, Canberra Australia, Keele United Kingdom, 2004.

[2] Noll, J., Beecham, S., \& Richardson, I. (2010). Global Software Development and Collaboration: Barriers and Solutions ACM Inroads, 1(3), 66-78.

[3] Jalali, S. and Wohlin, C. Agile Practices in Global Software Engineering - A Systematic Map. Dubinski, Y. and Milewski, A. eds. Global Software Engineering (ICGSE), 2010 5th IEEE International Conference on, IEEE, Princeton, NJ, 2010, 45-54.

[4] Kitchenham, B., Budgen, D. and Brereton, O. Using mapping studies as the basis for further research - A participant observer case study. Information and Software Technology, 53. 638-651. [5] Hooks, J. and F Corbett Jr Information literacy for off-campus graduate cohorts: Collaboration between a university librarian and a Master's of Education faculty. Library Review, 54 (4). 245 - 256. 